\title{
Limits of nature and advances of technology: What does biomimetics have to offer to aquatic robots?
}

doi:10.1533/abbi.2004.0028

\author{
F. E. Fish \\ Department of Biology, West Chester University, West Chester, PA 19383, USA
}

\begin{abstract}
In recent years, the biomimetic approach has been utilized as a mechanism for technological advancement in the field of robotics. However, there has not been a full appreciation of the success and limitations of biomimetics. Similarities between natural and engineered systems are exhibited by convergences, which define environmental factors, which impinge upon design, and direct copying that produces innovation through integration of natural and artificial technologies. Limitations of this integration depend on the structural and mechanical differences of the two technologies and on the process by which each technology arises. The diversity of organisms that arose through evolutionary descent does not necessarily provide all possible solutions of optimal functions. However, in instances where organisms exhibit superior performance to engineered systems, features of the organism can be targeted for technology transfer. In this regard, cooperation between biologists and engineers is paramount.
\end{abstract}

Key words: Biomimetics, drag reduction, aquatic locomotion, swimming, thrust production, evolution.

\section{INTRODUCTION}

The incorporation of novel structures and mechanisms from nature into the design and function of machines is being attempted through biomimetics. Biomimetics, or what was previously called bionics, attempts to produce engineered systems that possess characteristics, resemble, or function like living systems (Vogel 1998). The goal of biomimetics in the field of robotics is to use biological inspiration to engineer machines that emulate the performance of animals (Kumph and Triantafyllou 1998; Taubes 2000) particularly in instances where the animal's performance exceeds current mechanical technology.

It has been a long-standing idea that new technologies can be developed from nature (Fish 1998b; Vogel 1998). Animals served as the inspiration for various technological developments. Copying animals by the biomimetic approach attempts to seek common solutions from engineering and biology for increased efficiency and specialization (Vincent 1990). Because biological designs resulted from

\footnotetext{
Corresponding Author:

F. E. Fish

Department of Biology, West Chester University

West Chester, PA 19383, USA

Tel: 6104362460

Email: ffish@wcupa.edu
}

the evolutionary Darwinian process of "natural selection", it is considered that animals already performed the "costbenefit-analysis", optimizing particular designs for specific functions. Engineers may target the diverse morphological specializations exhibited by animals for technology transfer and effectively reduce the time of development of innovative technological solutions.

What are the limits to the biomimetic approach? Differences between engineered systems and animal systems are apparent. Engineered systems are relatively large in size, are composed of rigid materials, use rotation motors, and are controlled by computational systems with limited sensory feedback; in contrast, animals are generally small in size, are composed of compliant materials, use translational movements produced by muscles, and are controlled by complex neural networks with multiple sensory inputs. In addition, animals are functionally multifaceted (i.e. they move, feed, and reproduce). Animals must compromise optimal solutions for specialized functions to perform adequately rather than maximally (Katz and Jordan 1997; Webb 1997).

The potential for the development of new and superior technological designs for enhanced performance based on animal systems has been tantalizing, although elusive (Fish 1998b; Vogel 1998). Strict adherence to biological designs rarely produces any practical results and in some cases can impede the development of engineered systems (Vogel 1994, 1998; Fish, 1998b). For example, airplanes do not 
flap their wings like birds to simultaneously produce lift and thrust. Such a mechanism is impractical in modern aircraft due to limitations from scaling phenomena and the high speeds attained by commercial and military jets. As a result, the design of aircraft has advanced beyond the size and capabilities of birds for level flight. However, birds did serve as the inspiration for flight and the early development of wing design (Jakab 1990). Today, interest focuses on the ability of birds to perform complex aerial maneuvers. We cannot fly with the agility of birds, because we do not have the brains of birds to control the complex mechanical linkages, while appropriately sensing and regulating the airflow over the propulsor/control surfaces. In this regard, birds demonstrate superior performance to manufactured aircraft.

In aquatic systems, the emphasis on the biomimetic approach has been directed toward the use of locomotor specializations in animals associated with a reduction in energy input while swimming. For over 500 million years, fish and other animals were able to function and adapt to a fluid environment that is 800 times denser and 60 times more viscous than air. Machines that are required to work in the aquatic realm encounter the same physical forces as aquatic animals. Both natural and manufactured bodies are subjected to an environment where Archimedes Principle dominates and drag is a major hindrance to movement. Novel developments in engineered systems for operation in the aquatic environment are produced by both directly copying nature and by insight into independent convergence with animal designs (Bushnell and Moore 1991). By examination of processes by which the design of aquatic animals can be adapted to engineered systems, it may be possible to streamline the development of advanced technologies by biomimetics.

\section{CONVERGENCE OF DESIGN}

Convergence is a process in evolutionary biology whereby organisms from unrelated groups become more alike in appearance and function. This is typically the result of exposure to similar selection pressures over time. For aquatic animals and engineered systems that move rapidly through the water, the major determinate impinging on their shape and performance is drag. The magnitude of the drag is dependent on the density and viscosity of the fluid that determines the flow about the body.

It is no accident that modern submarines and dolphins possess the optimal shape for drag reduction of submerged bodies (Figure 1), although independently developed. Both possess fusiform body shapes that reduce the pressure drag. This streamlined profile is characterized by a rounded leading edge and slowly tapering tail. This design delays separation that occurs closer to the trailing edge, resulting in a smaller wake and reduced energy loss. Originally, submarine hulls were designed more as surface ships due to the limited amount of time that they could operate submerged. In 1953, the USS Albacore was built with a fusiform shape.
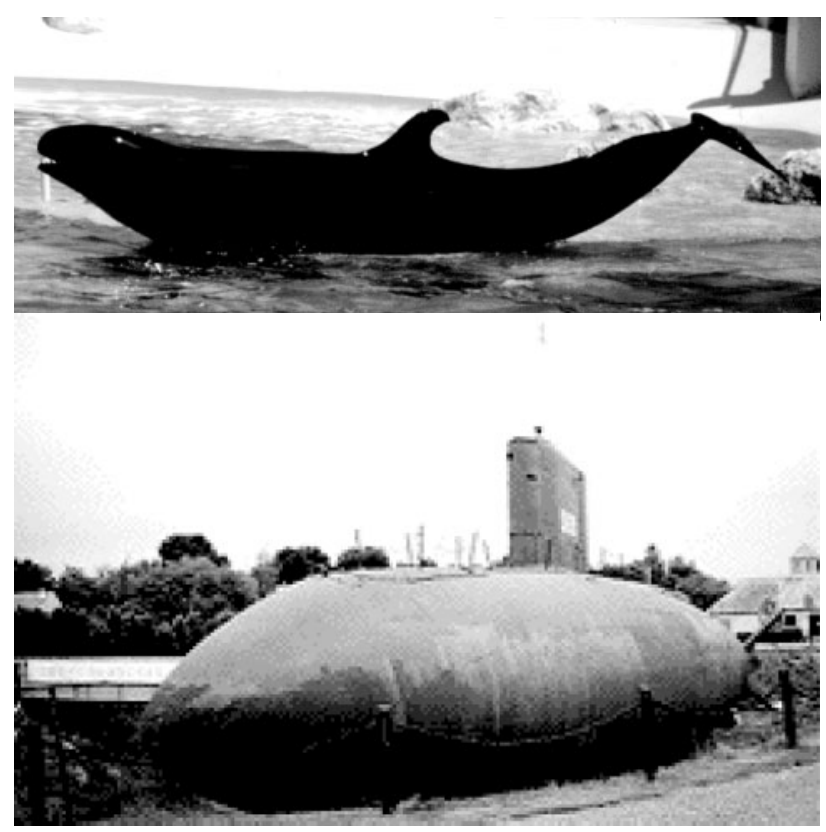

Figure 1 Fusiform shapes of dolphin (above) and submarine, USS Albacore (below).

The hull design of the Albacore was the forerunner for hulls used by nuclear submarines, which could travel submerged for extended periods. This streamlined hull made the Albacore the fastest and most maneuverable submarine of the time. Although the Albacore was likened to the shape of a fish, animals were not used as the basis for the design (Harris 1997).

The identification of animals as streamlined bodies with application to manufactured devices for drag was reported in the Renaissance. Between 1505 and 1508, Leonardo da Vinci wrote on the function of streamlined bodies in reducing drag (Anderson 1998). Da Vinci recognized the streamlined shape of a fish and demonstrated a similar design for the hull shape of ships. He argued that the fish could move through the water with little resistance, because its streamlined shape allowed the water to flow smoothly over the afterbody without prematurely separating.

In 1809, Cayley examined the streamlined body shapes of a trout and a dolphin as solids of least-resistance design (Gibbs-Smith 1962). Cayley's streamlined body for the fish is similar in design to low-drag airfoils. Application by Cayley of the natural design for a boat hull, however, did not meet with success (Vogel 1998). The rounded design was unstable with respect to roll, and low-drag did not occur. While appropriate for movement underwater, this shape is limited at the water surface.

Movement at the water surface requires a design with a sharp leading edge to reduce the formation of waves. Such a shape to reduce drag at the water surface is observed in the cross-sectional design of the toes of bats (Noctilio leporinus, Pizonyx vivesi) that are adapted for catching and eating fish (Fish et al. 1991). The bats use their echolocation to detect fish by ripples or breaks on the water surface and then drag their feet through the water to gaff the fish 
with their claws. The reversed fusiform cross-section reduces the additional drag components of wave and spray drag at the air-water interface (Hoerner 1965; Marchaj 1991). An analogous design is observed in the lower mandible of the black skimmer (Rhyncops nigra), which catches fish at the water surface with its beak (Withers and Timko 1977). Application of this mechanism to the struts of hydroplanes, however, is limited to rectilinear travel at the water surface, because the reversed fusiform design will incur premature separation with increased drag and loss of lift during turning maneuvers.

Many animals do swim at the water surface where they encounter the same forces and limitations as the standard displacement hulls of ships and yachts. As a displacement hull, surface swimming animals encounter high energy costs and limits to speed from gravitational waves of their own construction. As speed increases, the bow wave of displacement hulls increases in length. When the bow wave matches hull length, constructive interference between the bow and stern waves trap the hull in a trough preventing further increase in speed without the expenditure of a large amount of energy. This limit in speed is referred to as "hull speed".

Swimming speed for animals at the water surface is constrained by hull speed, which depends directly on the length of the body. Despite their small size, ducklings are able to exceed hull speed by the same mechanism used in marine engineering by replacing the displacement hull configuration with a planing hull (Aigeldinger and Fish 1995). The motion of a planing hull is described as "hydroplaning" or "skimming". With the hull inclined with a positive angle of trim, a positive pressure develops under the hull creating a vertical "dynamic lift" component, which at high speeds may be greater than buoyancy (Saunders 1957; Marchaj 1964). Ducklings use their high buoyancy and lift generated from the paddling hind feet to move in front of their own bow wave. The champion hydroplaning animals are the steamer ducks (Tachyeres spp.). Steamer ducks include three large, flightless species that hydroplane continuously on the water surface over distances of $1 \mathrm{~km}$ using their feet and wings (Livezey and Humphrey 1983; Aigeldinger and Fish 1995). The ducks reach speeds up to $6.67 \mathrm{~m} / \mathrm{s}$, which is over 13 hull lengths/s!

\section{COPYING}

In convergence, the resulting similarity between animals and engineered systems is merely coincidental. Indeed biologists typically note these similarities only after engineers developed structures with defined functions and described the principles by which they work. The similarity results from a finite number of possible solutions for dealing with particular forces that impinge on defined functions. Convergence is of limited utility in developing innovation as it is recognized in hindsight. However, convergence provides a natural validation of a similar engineered system, defines common environmental constraints, and helps to identify novel natural adaptations. Even subtle differences between natural features and their artificial analogs that can maximize performance may be exploited for improvement of already existing engineered designs.

Innovation by biomimetics, however, results from deliberate attempts to directly copy natural systems, once they were identified as functionally novel. The focus of this attention are features in organisms that convey superiority compared to human technology. The fantastic diversity of animals exhibits a multitude of natural innovations that can be used as a source of ideas and solutions for integration into engineered systems. For aquatic systems, the prime features to be copied are the abilities to function for prolonged periods of time while submerged, operate at high speeds, reduce energy consumption by increased propeller efficiency, maintain a high level of stability regardless of environmental perturbations, enhance maneuverability, and proceed with stealth.

An early attempt to copy nature was described by Borelli in 1680 (1960), who wrote on the design of a submarine that incorporated ideas based in part on hydrostatic control and propulsive systems of animals. The submarine would submerge by filling goatskin bags, located inside the submarine, through holes in the sides of the boat. Propulsion would be accomplished by oars projecting through the hull and fitted with watertight seals. When the submarine was on the bottom, the oars would push off the sandy substrate to move the boat along. In midwater, the oars would paddle like the feet of frogs or geese. During the rearward power stroke, a flexible paddle at the end of the oar would expand to work on a large mass of fluid. During the forward recovery stroke, the paddle would fold passively to reduce the frontal area and drag on the oar. However, Borelli considered that propulsion of the boat would be easier if a flexible oar were positioned at the stern emulating the motion of a fish tail. Despite the elaborate design for its time, it is doubtful that this early biomimetic experiment was successful (Harris 1997).

The investigation and application of special mechanisms for drag reduction by dolphins has been highly contentious (Gray 1936; Webb 1975; Fish and Hui 1991; Fein 1998). The controversy, known as "Gray's Paradox", was the result of the first attempt to evaluate swimming energetics in animals (Gray 1936; Webb 1975). Gray (1936) used a simple hydrodynamic model based on a rigid body to calculate drag power and applied it to a dolphin and a porpoise swimming at speeds of 10.1 and $7.6 \mathrm{~m} / \mathrm{s}$, respectively. The results indicated that the estimated drag power could not be reconciled with the available power generated by the muscles. For his calculations, Gray assumed that turbulent boundary flow conditions existed, because of the speed and size of the animals. Gray's resolution to the problem was that the drag on the dolphin was lower by maintenance of a fully laminar boundary layer. Gray proposed a mechanism to laminarize the boundary layer by accelerating the flow over the posterior half of the body. This mechanism was largely ignored in subsequent work, whereas, the basic 
premise that dolphins could maintain laminar boundary conditions remained and became the focus and justification of much of the work on dolphin hydrodynamics for the next 60 years (Kramer 1960a, b; Lang and Daybell 1963; Webb 1975; Aleyev 1977; Fish and Hui 1991; Fish 1998a).

The basic premise of Gray's Paradox, however, was flawed, because of potential errors in estimation of dolphin swimming speed and inconsistencies between dolphin swimming performance and data on muscle power outputs. Gray (1936) used a shipboard observation by a Mr. E. F. Thompson, who timed a dolphin with a stopwatch as it swam along the side of the ship (length = $41.5 \mathrm{~m}$ ) from stern to bow in $7 \mathrm{~s}$. If the dolphin was swimming close enough to utilize the wave system of the ship, its speed may have been artificially enhanced and energetic effort reduced due to free-riding behaviors (Lang 1966; Williams et al. 1992). Later, Gray (1968) used speed data of $10.3 \mathrm{~m} / \mathrm{s}$ for a $9 \mathrm{~s}$ effort from Stevens (1950), but the dolphin also was swimming close to the ship.

More important than the actual speed, the observations of the dolphin swimming speeds were for sprints (7-9 s) and Gray used measurements for muscle power output of sustained performance ( $3-5 \mathrm{~min}$ ) by human oarsmen (Henderson and Haggard 1925). Muscle performance is a function of the type of muscle fibers stimulated during an activity. Fast glycolytic (FG) fibers are adapted for short burst activities with high power output and very high intrinsic speed of shortening; whereas, slow oxidative (SO) fibers are slow contracting and are suitable for slow, sustained activity (Alexander and Goldspink 1977). The peak power outputs are 2.6-3 times greater for FG than SO fibers (Barclay et al. 1993; Askew and Marsh 1997). Both FG and SO fibers are found in the musculature of cetaceans (Ponganis and Pierce 1978; Suzuki et al. 1983 ; Bello et al. 1985). FG fibers are fueled primarily by anaerobic metabolism and $\mathrm{SO}$ fibers use primarily aerobic metabolism. Depending on the type of metabolic pathway, anaerobic metabolism has a maximum metabolic power output 2-17 times greater than aerobic metabolism (Hochachka 1991).

If the dolphins were truly swimming at $10.1 \mathrm{~m} / \mathrm{s}$ without interference from the ships (Gray 1936; Stevens 1950), the short duration of the activity indicates the use of FG fibers and higher power outputs (Webb 1975; Fish and Hui 1991). Gray (1936) calculated muscle power outputs of $14 \mathrm{~W} / \mathrm{kg}$ for a dolphin with a low-drag laminar boundary layer and $122 \mathrm{~W} / \mathrm{kg}$ with a high-drag turbulent boundary layer, respectively. With anaerobic contributions, Tursiops truncatus could generate an estimated $110 \mathrm{~W} / \mathrm{kg}$ (Weis-Fogh and Alexander 1977).

Gray's Paradox was invigorated by the work of Kramer (1960a, b, 1965). Kramer claimed that a laminar boundary layer without separation could be achieved at high Reynolds number (Re; ratio of inertial to viscous forces) by artificially increasing the viscosity at the body surface (Webb 1975).
Kramer (1960a) coated a torpedo with an artificial skin based on the skin of a dolphin. The dolphin integument is composed of a smooth, hairless epidermal surface forming an elastic membrane (Kramer 1960a; Aleyev 1977) and anchored to the underlying dermis with its blubber layer by longitudinal dermal crests with rows of papillae, which penetrate the lower epidermis (Kramer 1960b, 1965; Sokolov et al. 1969; Yurchenko and Babenko 1980; Haun et al. 1983). Kramer's analogous skin was composed of a heavy rubber diaphragm supported by rubber studs with the intervening spaces filled with a viscous silicone fluid (Kramer 1960a, b; 1965). The diaphragm would be sensitive to pressure changes and transmit the pressure oscillations below to the viscous fluid. The fluid would flow beneath the diaphragm to absorb part of the turbulent energy. It was hypothesized that the coating would dampen out perturbations in the flow and prevent or delay transition. When a towed body was coated with the artificial skin, anterior of the maximum thickness, a $59 \%$ reduction in drag was achieved at $\operatorname{Re}=15 \times 10^{6}$ compared to a rigid reference model with fully turbulent flow. These results exposed the "dolphin's secret" and provided a resolution to Gray's Paradox (Kramer 1960b).

The structure of Kramer's skin coating and the skin and underlying blubber of dolphins is both structurally and mechanically different. Despite these differences, research on compliant coatings was accelerated during the 1960s (Aleyev 1977; Fish and Hui 1991; Riley et al. 1988). However, attempts to verify Kramer's results subsequently failed (Landahl 1962; Riley et al. 1988). Furthermore, experiments on live dolphins and a review of the available literature on dolphin swimming performance showed no evidence for drag reduction from special mechanisms (Lang and Daybell 1963; Fish and Hui 1991; Fish and Rohr 1999).

A more successful application of copying natural designs for drag reduction was found for riblets. The development of riblets to reduce turbulent skin friction came in part from the study of shark scales or dermal denticles (Walsh 1990). Riblets are streamwise microgrooves that act as fences to break up spanwise vortices, and reduce the surface shear stress and momentum loss. Fast swimming sharks have scales that are different from other sharks. These scales have flat crowns and sharp ridges oriented longitudinally with rounded valleys (Pershin et al. 1976; Reif 1978, 1985; Reif and Dinkelacker 1982). Although the ridges are discontinuous due to the distribution of the scales, a 7-8\% drag reduction is possible as measured for continuous riblets (Reidy 1987; Walsh 1990). Riblets were considered for use on aircraft. Because the fine microgrooves are prone to fouling, riblets are limited to short duration usage. However, riblets were applied to the hull of the Stars \& Stripes, the 1987 America's Cup winner. Riblets were believed to reduce drag from skin friction by $2-4 \%$ (Letcher $e t$ al. 1987). Recently, Speedo developed a competitive, wholebody swimsuit called Fastskin ${ }^{\mathrm{TM}}$ that is based on shark scales. 

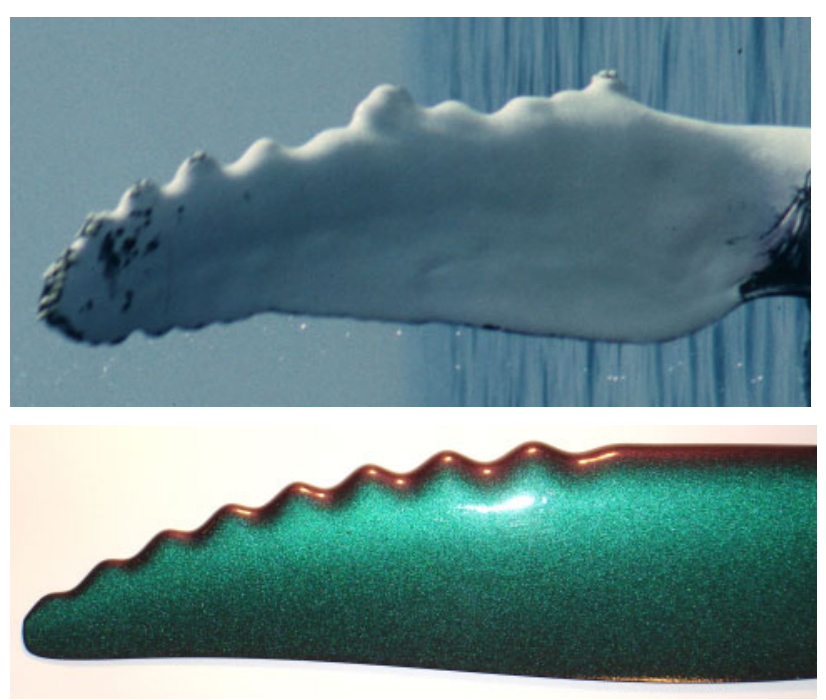

Figure 2 Actual (top) and model (bottom) flippers of humpback whale showing leading edge tubercles. Photograph of humpback whale flipper courtesy of W. W. Rossiter.

Humpback whale flippers exhibit a curved plan form, large aspect ratio, and high mobility, especially when compared with other whale species (Figure 2). These flippers closely resemble the $21 \%$ thick, low-drag NACA $63_{4}-021$ wing in cross-section. Humpback whale flippers also display leading edge tubercles, essentially sinusoidal bumps facing into the free stream flow that alter the fluid flow over these wing-like flippers (Bushnell and Moore, 1991; Fish and Battle, 1995). Humpback whales are the only cetaceans with tubercles, and the only baleen whale that relies on maneuverability to capture prey (Fish and Battle, 1995). Specifically, humpback whales use their flippers to achieve tight circles while corralling and engulfing prey. Tubercles could provide an advantage in maneuverability and prey capture.

Recently, analysis by computational methods and wind tunnel testing showed that the presence of leading edge tubercles on wings increase useful force production while simultaneously reducing parasitic forces and delaying stall (Watts and Fish, 2001; Miklosovic et al., 2004). The stall angle was increased by $40 \%$ for a model wing with leading edge tubercles compared to a wing with straight leading edge (Miklosovic et al., 2004). A number of possible fluid dynamic mechanisms could be responsible for improved performance, including stall delay through either vortex generation or modification of boundary-layer flow, or increase in effective span by reduction of both spanwise flow and strength of tip vortex. Few other passive means of altering fluid flow around a wing can both increase lift and reduce drag at the same time.

The potential for enhanced performance by emulating nature also focuses on propulsive systems. The thrust performance of fish and dolphin tails is considered superior to screw propellers (Pettigrew 1893; Peterson 1925; Triantafyllou and Triantafyllou 1995). Early versions

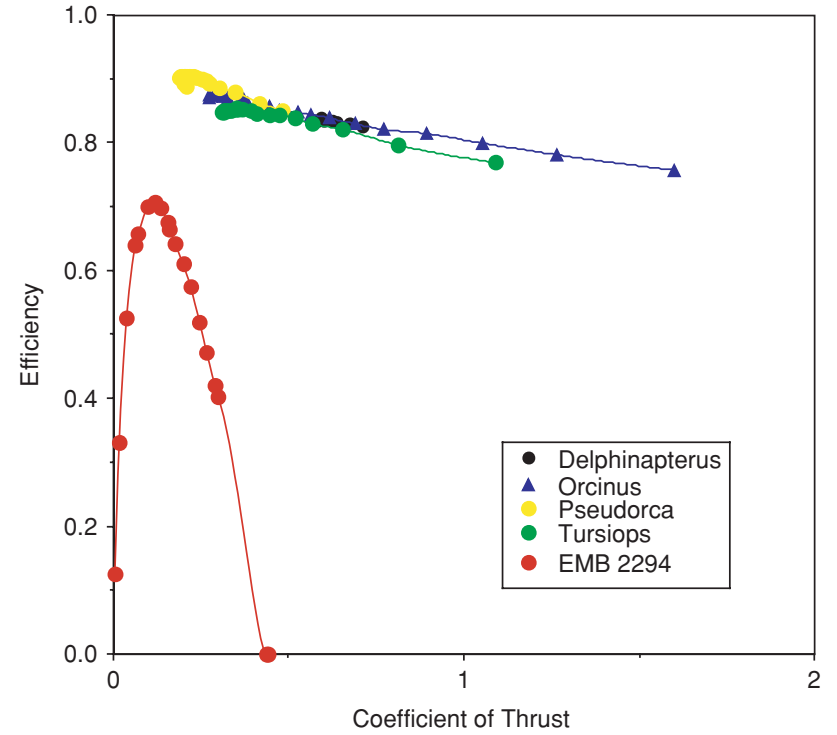

Figure 3 Comparison of relationships of propulsive efficiency and thrust coefficient for four species of small whales and a typical marine propeller. Data for the whales was obtained from Fish (1998) and data for the propeller was from Saunders (1957).

of propellers could not change their pitch with speed, because of the fixed nature of the blades. This was believed to limit effectiveness over the speed range of the propeller due to caviation. The oscillatory motions of flexible-bodied fish and dolphin were considered to be able to adjust to velocity changes and maintain effective thrust production over a large speed range (Pettigrew 1893; Saunders 1951, 1957). Figure 3 shows that the oscillatory flukes of dolphins' function at higher efficiencies over a greater range of thrust coefficients compared to a standard propeller. Conventional marine propellers operate at mechanical efficiencies of about 70\% (Larrabee 1980), whereas high-performance swimmers, such as tuna, dolphins, and seals, are able to produce efficiencies of over $80 \%$ (Webb 1975; Fish and Rohr 1999). The emulation of oscillatory movements for aquatic locomotion has lead to the development of exoskeletons, fins and submarines using humanpowered propulsion (Figures 4, 5 and 6; Neuhaus et al., 2004).

Presently, biomimetic robots are being produced that emulate the propulsive systems of fish, dolphins, and seals (Figure 7) (Triantafyllou and Triantafyllou 1995; Anderson and Kerrebrock 1997, 1999; Bandyopadhyay and Donnelly 1997; Bandyopadhyay et al. 1997; Kato 1998, 1999; Kumph and Triantafyllou 1998; Wolfgang et al. 1998; Nakashima and Ono 1999; Taubes 2000). A primary focus of this research is based on the idea that these animals can enhance thrust production and increase efficiency by controlling vorticity shed from the body and propulsors (Ahlborn et al. 1991; Gopalkrishnan et al. 1994; Triantafyllou et al. 1996; Bandyopadhyay and Donnelly 1997; Anderson et al. 1998; Wolfgang et al. 1999). 


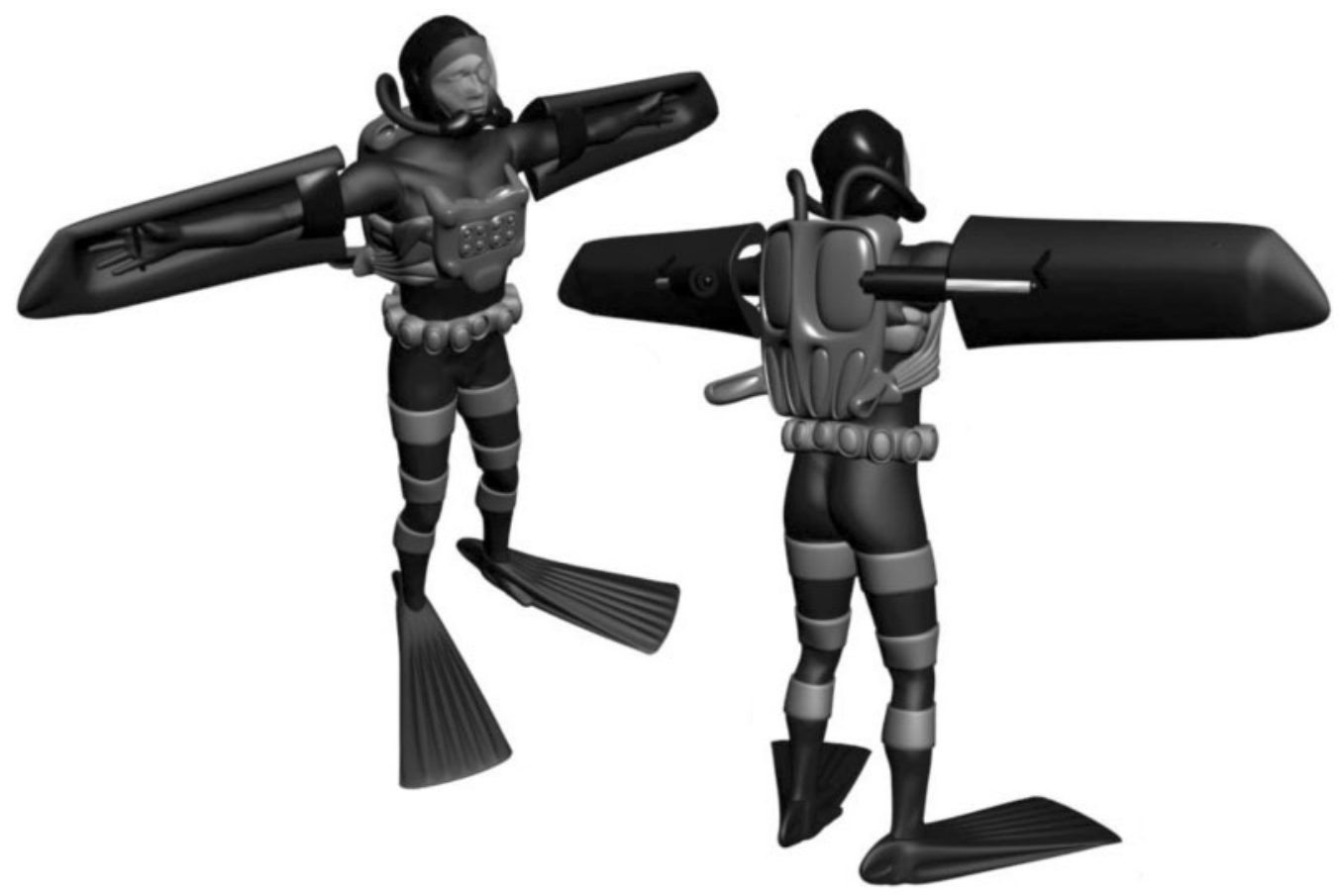

Figure 4 Human exoskeleton for biomimetic propulsion (Neuhaus et al. 2004). The human diver is envisioned to swim by flapping motion, which is similar to the propulsive strokes of certain fish, penguins, and sea lions. Drawing courtesy of P. D. Neuhaus.

Ahlborn et al. (1991) developed the vortex excitation/ destruction model. In the model, a starting vortex forms before being acted on by the fin as it quickly reverses. This action produces new vortices on the opposite side of the fin, which gain strength at the expense of the primary vortex. Higher power is achieved (Ahlborn et al. 1991). This mechanism is particularly applicable to starts from rest. Experimental manipulation of vorticity shed from an oscillating hydrofoil by interaction with an anteriorly generated vortex showed optimal efficiency at frequencies of maximum amplification of the propulsive jet (Triantafyllou et al. 1993).

Using digital particle image velocimetry (DPIV), the development of vorticity along the sides of an undulating fish was demonstrated (Wolfgang et al. 1999). This vorticity developed in a manner similar to flow along an undulating plate. The bound vorticity was conducted toward the trailing edge of the caudal fin. The bound vortices combined as they were being shed into the wake to produce an amplified vortex. The next set of vortices shed into the wake had the opposite rotation. This produced a pair of counter-rotating vortices and a thrust jet. Continuous vortex shedding produces a wake with the thrust-type, reverse Karman vortex street (Weihs 1972; McCutchen 1977; Müller et al. 1997; Wolfgang et al. 1999). The interaction of vorticity generated along the body and shed at the caudal fin conformed to the mechanism discussed by Gopalkrishnan et al. (1994). A similar pattern of vorticity was observed for fish executing a turn (Wolfgang et al. 1999). It was postulated that this mechanism of propulsion was dependent on active control involving coordination of the body undulation and caudal fin motion (Wolfgang et al. 1999).

Use of vorticity generated along the body to enhance wake structure is limited in high-performance thunniform swimmers. These animals are relatively stiff anteriorly, have nearly circular or elliptical cross-sections, and display extreme narrow necking (Webb 1975; Fish and Hui 1991; Fish and Rohr 1999). These morphological features should not promote the production and conduction of bound vorticity along the body. However, the heaving and pitching motions of the relatively stiff, high-aspect ratio caudal propulsor could produce leading edge vortices, which would impact the wake structure (Anderson et al. 1998). The development of leading edge vortices from dynamic stall was experimentally demonstrated to produce high lift forces (Ellington et al. 1996; Dickinson et al. 1999). The leading edge vortex would interact with the trailing edge vortex to produce thrust. This vorticity control is the principle mechanism for high efficiency (Anderson et al. 1998). Anderson et al. (1998) demonstrated propulsive efficiencies of over $85 \%$ for a rigid flapping foil. More recent research shows that chordwise flexibility of a flapping foil can increase the efficiency by up to $36 \%$ over a rigid foil (Prempraneerach et al. 2003). This flexibility is present already in the oscillating propulsors of animals and may similarly enhance propulsive efficiency.

Experiments with the biologically-inspired RoboTuna showed that under a particular set of kinematic conditions, the swimming robot could reduce its drag in excess of $70 \%$ compared to the same body towed straight and rigid 

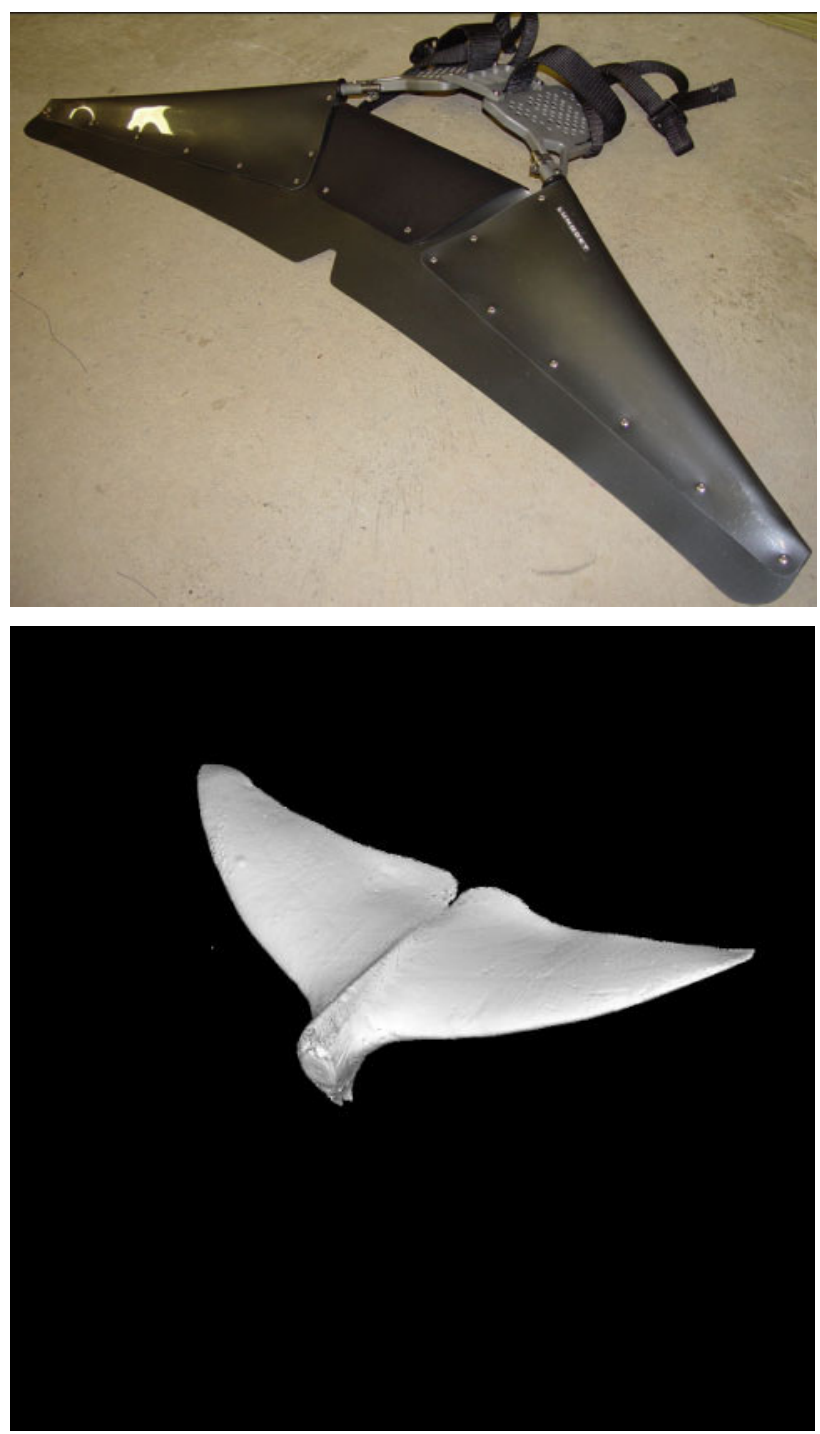

Figure 5 Biomimetic monoflipper for dolphin-like swimming (top) and three-dimensional reconstruction of dolphin flukes from CT scans (bottom). Photograph of monoflipper courtesy of T. Ciamillo.

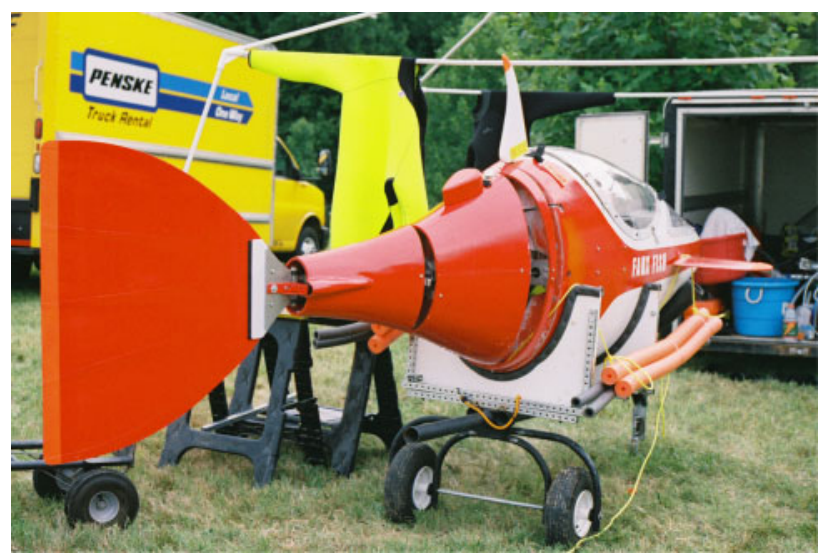

Figure 6 Human-powered submarine using lateral caudal undulations of a flexible and jointed tail fin.

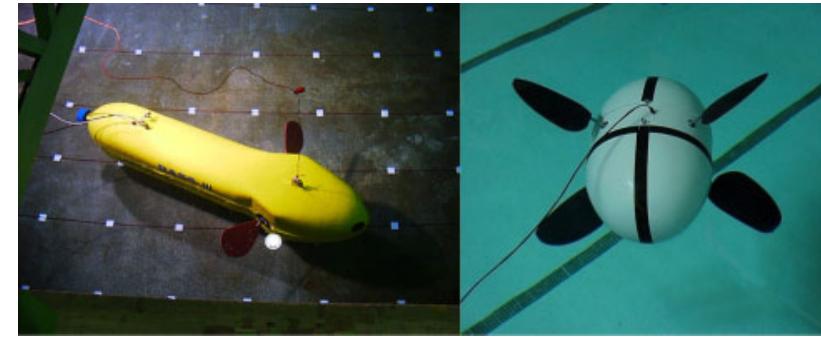

Figure 7 Biomimetic robots Bass3 (right) and PilotFish (left). Image of Bass 3 was provided courtesy of $\mathrm{N}$. Kato and image of PilotFish was courtesy of Nekton Research, LLC.

(Barrett et al. 1999). The conditions, which produced this reduction in drag, deviated from the kinematics found in living thunniform swimmers. In particular, the lateral excursion of the caudal propulsor was $12 \%$ of body length (Barrett et al. 1999), whereas the typical excursion of the caudal propulsor in animals is approximately $20 \%$ of body length (Webb 1975; Dewar and Graham 1994; Fish 1998a; Gibb et al. 1999). Deviation from the optimal settings of kinematic parameters for RoboTuna resulted in a drag augmentation of 300\% (Barrett et al. 1999).

Biologists and engineers considered that the development of more economical propulsive systems was possible from energy storage by spring-like mechanisms. Tendons, muscle and blubber have been proposed as possible structures for elastic storage in swimming animals (Wainwright et al. 1985; Bennett et al. 1987; Alexander 1988; Blickhan and Cheng 1994; Pabst 1996; Lindstedt et al. 2002). Direct evidence of energy savings by spring-like mechanisms has not been forthcoming (Bennett et al. 1987) and may be limited due to the dampening nature of water. However, models using oscillating foils demonstrate reduced energy costs (Harper et al. 1998; Nakashima and Ono 1999; Murray and Howle 2003).

Replication of the turning performance of aquatic animals as an indication of maneuverability was attempted. Autonomous fishlike robots with turning capabilities were constructed with jointed bodies (Anderson and Kerrebrock 1997; Kumph and Triantafyllou 1998). Utilizing vorticity control, these flexible, biomimetic fish showed increased maneuvering capabilities compared to conventional unmanned underwater vehicles. Biomimetic fish can turn at a maximum rate of $75^{\circ} / \mathrm{s}$, whereas conventional rigid-bodied robots and submarines turn at approximately 3-5\% (Miller 1991; Anderson and Kerrebrock 1997). Success was attained also in matching the turn radius $(47 \%$ of body length) with the group of fish (tuna) on which the robot's design was based (Blake et al. 1995; Anderson and Kerrebrock 1997). Further refinements and use of alternate animal models could result in improved maneuverability. Various aquatic animals display turn rates up to $5509^{\circ} / \mathrm{s}$, centripetal accelerations up to $24.5 \mathrm{~g}$, and minimum turn radii of 24\% of body length and lower (Daniel and Webb 1987; Fish 1999; Gerstner 1999). 


\section{LIMITATIONS OF BIOMIMETICS}

Aquatic animals have existed in an environment that they mastered, for millions of years. It is viewed that over this time evolution (descent with modification) through the Darwinian process of "natural selection" fostered improvements in design, which culminated in adaptations for the survival of these organisms by enhanced levels of performance. Evolution is perceived to act as a natural laboratory. Given a time scale that is geologic, virtually all the possible permutations of these natural experiments could be attempted. Because natural selection chooses from a wide range of design and performance possibilities as dictated through the genetic code and functional demand of the local environment, a variety of possible solutions to engineering problems are potentially available. However, the laws of physics and the physical properties of environment and structural materials available to biological forms impose constraints on evolution (Alexander 1985). Possible structures and processes that potentially could benefit an organism are not all available. Wheels are not found in animals, despite their ubiquity in manufactured devices and their obvious benefit to energy economy in locomotion. Animals move through forceful contraction of the muscles transmitted to a jointed skeleton by tendonous connections. Therefore, biological systems suffer lower efficiency due to periodic accelerations over a propulsive cycle. Large animals are unable to produce high rates of acceleration, because as size increases the ability of the muscles to generate stresses relative to inertial forces decreases (Webb 1988).

Evolution is not conscious or predictive. Evolution by the theory of natural selection is a response to changing environments. The biotic and abiotic environments of the time that a new design evolves dictate its selection without anticipation for potential future purpose and effectiveness. Indeed, it is difficult if not impossible for any design to be optimized. The environment is nearly always changing. This change produces a nonequilibrium state, which places design criteria in a state of constant flux (Lauder 1991). Both superior and poor designs with respect to present time may be lost if they did not function adequately in past environments or if they were accidentally lost due to chance events. Use of the term 'design' in a biological sense is simply an indication of the linkage between the structure and function of a characteristic possessed by an organism. For biologists, design does not infer construction or organization of an organism's feature toward a specific goal (Gosline 1991).

Another restriction to design is that animals evolved along lines of common descent with shared developmental patterns. Radical redesigns are not permitted to expedite enhancing performance; instead, it is existing designs that are modified (Vogel 1998). Within a given lineage, phenotypic change is expressed as variations on a theme. Design is constrained by the evolutionary history of an organism. Swimming in whales would be more efficient if these animals remained submerged like fish, because drag increases due to the formation of waves as a body moves in close proximity to the water surface; however, their common evolutionary history with other air-breathing mammals requires that they periodically return to the water surface to fill their lungs despite increased energy cost.

Animals are multitasking entities. While machines can be designed for a single function, animals must endure compromises in their designs to perform multiple and sometimes antithetical functions. Increased performance by one feature that benefits an organism for a particular function may handicap the organism with respect to another function. Depending on the local environment and immediate selection forces, genetic linkages between traits and pleiotrophic effects can produce changes in one characteristic that produce a correlated effect in other characteristics (Lauder 1991). In total, the organism as a mosaic of integrated structures and functions may achieve evolutionary success (i.e. survive and reproduce), but not perform optimally for any specific function.

Efficiency is an important factor that received attention from engineers wishing to employ a biomimetic approach to the propulsive systems of marine robots (Triantafyllou and Triantafyllou 1995; Anderson et al. 1998). Oscillatory mechanisms employing stiff, high-aspect ratio hydrofoils, as displayed by tuna, dolphins, and seals, were targeted for technology transfer for increased efficiency. The assumption is that any mechanism that allows for increased energy efficiency can provide an important evolutionary advantage to an animal. However, natural selection does not necessarily act on efficiency. Efficiency is measured as the ratio of the useful work performed to the total work produced. The oscillating hydrofoil propulsor of an animal performs useful work by generating thrust from the acceleration of water in the opposite direction to the animal's progression; whereas, the total work performed is the sum of the useful work and the work on the water in the transverse direction. Because the work is performed on the environment to effect locomotion, this is the external work. The muscles to accelerate and decelerate individual body segments perform internal work. The total energy cost for locomotion will be high from the internal work associated with an oscillatory system, despite the high efficiency of the oscillating hydrofoil with respect to external work.

Natural selection will act on energy economy rather than efficiency. Economy refers to the total cost of a given task, and thus considers rate of energy consumption with respect to the available supply of energy and the rate at which energy can be resupplied. For an animal, swimming at a high speed is more efficient than swimming at a low speed, but the rate at which energy is consumed to swim fast is disproportionately higher than swimming slowly. High speeds cannot be maintained for extended periods as the total energy reserves are depleted rapidly and new energy resources cannot be mobilized quickly enough. Rapid swimming is limited to those instances where survival of 
the animal is at stake. Escape by prey and bursts by predators demand large and rapid energy expenditures without concern about efficiency.

For routine swimming speeds, energy economy determines the time and distance that can be traversed. Animals will swim at their optimal speed. Optimal speed is the speed at which the total cost of energy per unit distance is minimal (Webb 1975). Optimal speed is typically found at intermediate swimming speeds for animals. The cost of maintaining the metabolism is high relative to the locomotor costs at low speeds and the cost to traverse a given distance is high. At speeds greater than the optimum, locomotor costs increase exponentially, driving up the cost per distance, although the time to cover that distance is decreased. Migratory species and animals that travel long distances for prey that has a patchy distribution will swim at speeds near optimal.

\section{CONCLUSIONS}

The technology associated with the development of robots is becoming more dependent on biomimetics and biologically inspired designs. As engineers move from the world of large, stiff, right-angled pieces of metal to one of small, compliant, curved-surface pieces of heterogeneous parts, nature will become a more influential teacher. Animal systems hold promise for improved performance by machines in the aquatic realm (Shaw 1959; Bushnell 1998). As matters of energy economy and greater locomotor performance are desired in engineered systems, imaginative solutions from nature may serve as the inspiration for new technologies. Enhanced propulsion for engineered systems may be possible by biomimetic mechanisms involved with oscillating propellers, flexible wings, boundary layer stabilization, laminar flow maintenance, and active vortex control. In addition, natural propulsive systems can be self-stabilizing and self-correcting. The potential benefits from biological innovations applied to manufactured systems operating in water are high speeds, reduced detection, energy economy, and enhanced maneuverability.

Progress in technologies concerned with aquatic locomotion comes from discovery and refinement of new designs. New insights into aquatic propulsion by animals will permit the collection of information that can be exploited for the development of advanced technologies. These insights include an interpretation of the unsteady nature of animal movement, measurement of movement in two and three dimensions, the use of computational methods that model both the animal's movement and its effect on the fluid surrounding, physiological and biomechanical studies of locomotor tissues in relation to thrust production and energy recycling, and analysis of the use of appendages in thrust production, trajectory control, and stability (Lauder and Long 1996).

Perhaps the process of evolution can be used directly in a biomimetic approach. By using high-speed computers, an initial design can be subjected to various combinations of selection pressures. As the design "evolves", each configuration could be tested virtually to assess its level of performance. Like evolution, this would be an open-ended process and new selection pressures could be introduced to produce alternative branching pathways. Designs considered as useful could be manufactured without a haphazard development phase and without a prolonged time of testing prototypes.

In comparison to engineers who can limit variables in their systems, biologists examine systems of complexity. These biological systems interact with their environments in a multitude of behavioral and morphological pathways and are composed of structural elements for which the physical characteristics have not been fully described. Nature, therefore, retains a store of untouched knowledge, which would be beneficial to engineers. In an everincreasing era of specialization and a proliferation of information, it is nearly impossible to find novel solutions from disparate fields other than one's own. Thus, collaborations become essential and the union between biologists and engineers is critical to biomimetics (Shaw 1959).

\section{ACKNOWLEDGMENTS}

I would like to express my appreciation to Naomi Kato and Yoshihiro Suzuki for their sponsorship of the symposium for which this paper was originally presented. This paper is based in part on research performed with support from the Office of Naval Research (ONR) and Defense Advanced Research Projects Agency (DARPA).

\section{REFERENCES}

Ahlborn B, Harper DG, Blake RW, Ahlborn D, Cam M. 1991. Fish without footprints. 7 Theor Biol, 148:521-533.

Aigeldinger TL, Fish FE. 1995. Hydroplaning by ducklings: Overcoming limitations to swimming at the water surface. J Exp Biol, 198:1567-1574.

Alexander RMcN, Goldspink G. 1977. Mechanics and Energetics of Animal Locomotion. London: Chapman \& Hall.

Alexander RMcN. 1985. The ideal and the feasible: Physical constraints on evolution. Biol f Linn Soc, 26:345-358.

Alexander RMcN. 1988. Elastic mechanisms in animal movement. Cambridge: Cambridge University Press.

Aleyev YG. 1977. Nekton. The Hague: Junk.

Anderson JD. 1998. A history of aerodynamics. Cambridge: Cambridge University Press.

Anderson JM, Kerrebrock PA. 1997. The vorticity control unmanned undersea vehicle - An autonomous vehicle employing fish swimming propulsion and maneuvering In Tenth International Symposium on Unmanned Untethered Submersible Technology: Proceedings of the Special Session on Bio-Engineering Research Related to Autonomous Underwater Vehicles. 1997. Lee, NH: Autonomous Undersea Systems Institute. p. 189-195.

Anderson JM, Kerrebrock PA. 1999. The vorticity control unmanned undersea vehicle (VCUUV) performance results, In Eleventh International Symposium on Unmanned Untethered Submersible Technology. 1999. Lee, NH: Autonomous Undersea Systems Institute. p. 360-369. 
Anderson JM, Streitlien K, Barrett DS, Triantafyllou MS. 1998 Oscillating foils of high propulsive efficiency. 7 Fluid Mech, 360:41-72.

Askew GN, Marsh RL. 1997. The effects of length trajectory on the mechanical power output of mouse skeletal muscles. $\mathcal{F}$ Exp Biol, 200:3119-131.

Bandyopadhyay PR, Donnelly MJ. 1997. The swimming hydrodynamics of a pair of flapping foils attached to a rigid body In Tenth Internat. Symp. Unmanned Untethered Submersible Tech.: Proc. Sp. Ses. Bio-Eng Res. Related to Autonomous Underwater Vehicles.1997. Lee, NH: Autonomous Undersea Systems Institute. p. 27-43.

Bandyopadhyay PR, Castano JM, Rice JQ, Philips RB, Nedderman WH, Macy WK. 1997. Low-speed maneuvering hydrodynamics of fish and small underwater vehicles. Trans ASME, 119:136-144.

Barclay CJ, Constable JK, Gibbs CL. 1993. Energetics of fast-twitch and slow-twitch muscles of the mouse. 7 Physiol, 472:61-80

Barrett DS, Triantafyllou MS, Yue D KP, Grosenbaugh MA, Wolfgang MJ. 1999. Drag reduction in fish-like locomotion. 7 Fluid Mech, 392:183-212.

Bello MA, Roy RR, Martin TP, Goforth HW Jr., Edgerton VR. 1985. Axial musculature in the dolphin (Tursiops truncatus): Some architectural and histochemical characteristics. Mar Mamm Sci, 1:324-336.

Bennett MB, Ker RF, Alexander R McN. 1987. Elastic properties of structures in the tails of cetaceans (Phocoena and Lagenorhynchus) and their effect on the energy cost of swimming. 7 Zool Lond, 211:177-192.

Blake RW, Chatters LM, Domenici P. 1995. Turning radius of yellowfin tuna (Thunnus albacares) in unsteady swimming manoevres. 7 Fish Biol, 46:536-538.

Blickhan R, Cheng JY. 1994. Energy storage by elastic mechanisms in the tail of large swimmers - a re-evaluation. 7 Theor Biol, 168:315-321.

Borelli GA. 1960. De motu animalium pars (The Movement of Animals, translated by P. Maquet) Berlin: Springer-Verlag.

Bushnell DM, Moore KJ. 1991. Drag reduction in nature. Ann Rev Fluid Mech, 23:65-79.

Bushnell DM. 1998. Drag reduction "designer fluid mechanics"Aeronautical status and associated hydrodynamic possibilities (an "embarrassment of technical riches") In Proceedings of the International Symposium on Seawater Drag Reduction 1998. J. C. S. Meng, Ed. Newport, RI.

Daniel TL, Webb PW. 1987. Physics, design, and locomotor performance In Comparative Physiology: Life in Water and on Land. 1987. P Dejours, L Bolis, C R Taylor, E R Weibel. New York: Liviana Pr. p. 343-369.

Dewar H, Graham JB. 1994. Studies of tropical tuna swimming performance in a large water tunnel. III. Kinematics. $\mathcal{F}$ Exp Biol, 192:45-59.

Dickinson MH, Lehmann FO, Sane SP. 1999. Wing rotation and the aerodynamic basis of insect flight. Science, 284 1954-1960.

Ellington CP, van den Berg C, Willmott AP, Thomas ALR. 1996. Leading-edge vortices in insect flight. Nature, 384:626-630.

Fein JA. 1998. Dolphin drag reduction: Myth or magic? In Proceedings of the International Symposium on Seawater Drag Reduction, J. C. S. Meng, Ed. Newport, RI.1998 p. $429-433$.
Fish FE, Blood BR, Clark BD. 1991. Hydrodynamics of the feet of fish-catching bats: Influence of the water surface on drag and morphological design. 7 Exp Zool. 258:164-173.

Fish FE, Hui CA. 1991. Dolphin swimming - a review. Mamm Rev, 21:181-195.

Fish FE, Battle JM. 1995. Hydrodynamic design of the humpback whale flipper. 7 Morph, 225:51-60.

Fish FE, Rohr J. 1999. Review of dolphin hydrodynamics and swimming performance. SPAWARS System Center Technical Report 1801, San Diego, CA.

Fish FE. 1998a. Comparative kinematics and hydrodynamics of odontocete cetaceans: Morphological and ecological correlates with swimming performance. 7 Exp Biol, 201:2867-2877.

Fish FE. 1998b. Imaginative solutions by marine organisms for drag reduction. In Proceedings of the International Symposium on Seawater Drag Reduction, JCS Meng, Ed. Newport, RI. p. 443-450.

Fish FE. 1999. Performance constraints on the maneuverability of flexible and rigid biological systems In Eleventh International Symposium on Unmanned Untethered Submersible Technology, Lee, NH: Autonomous Undersea Systems Institute. 1999. p. 394-406.

Gerstner CL. 1999. Maneuverability of four species of coral-reef fish that differ in body and pectoral-fin morphology. Can $\mathcal{F}$ Zool, 77:1102-1110.

Gibb AC, Dickson KA, Lauder GV. 1999. Tail kinematics of the chub mackerel Scomber japonicus: Testing the homocercal tail model of fish propulsion. 7 Exp Biol, 202:2433-447.

Gibbs-Smith CH. 1962. Sir George Cayley's aeronautics 1796-1855. London: Her Majesty's Stationery Office.

Gopalkrishnan R, Triantafyllou MS, Triantafyllou GS, Barrett D. 1994. Active vorticity control in a shear flow using a flapping foil. 7 Fluid Mech, 274:1-21.

Gosline JM. 1991. Efficiency and other criteria for evaluating the quality of structural biomaterials In Efficiency and Economy in Animal Physiology. 1991 R. W. Blake, Ed. Cambridge: Cambridge University Pr. p. 43-64.

Gray J. 1936. Studies in animal locomotion VI. The propulsive powers of the dolphin. 7 Exp Biol, 13:192-199.

Gray J. 1968. Animal Locomotion. London: Weidenfeld and Nicolson.

Harper KA, Berkemeier MD, Grace S. 1998. Modeling the dynamics of spring-driven oscillating-foil propulsion. IEEE $\mathcal{F}$ Ocean Eng, 23:285-296.

Harris B. 1997. The Navy Times book of submarines: A political, social, and military history. New York: Berkley.

Haun JE, Hendricks EW, Borkat FR, Kataoka RW, Carder DA, Chun NK. 1983. Dolphin hydrodynamics: Annual report FY 82. NOSC Tech. Rep. 935.

Henderson Y, Haggard HW. 1925. The maximum of human power and its fuel. Amer $\mathcal{F}$ Physiol, 72:264-282.

Hochachka PW. 1991. Design of energy metabolism, In Environmental and metabolic animal physiology. 1991 C. L. Prosser, Ed., New York: Wiley-Liss Pr. p. 325-351.

Hoerner SF. 1965. Fluid-dynamic drag. Brick Town, New Jersey: Published by author.

Jakab PL. 1990. Visions of a flying machine. Washington: Smithsonian Institution Press.

Kato N. 1998. Locomotion by mechanical pectoral fins. 7 Mar Sci Technol, 3:113-121.

Kato N. 1999. Hydrodynamic characteristics of a mechanical pectoral. F Fluid Eng, 121:605-613. 
Katz SL, Jordan CE. 1997. A case for building integrated models of aquatic locomotion that couple internal and external forces In Tenth International Symposium on Unmanned Untethered Submersible Technology. Durham, NH, 1997, pp. 135-152.

Kramer MO. 1960. Boundary layer stabilization by distributed damping. 7 Amer Soc Nav Eng, 72:25-33.

Kramer MO. 1960. The dolphins' secret. New Sci, 7:1118-1120.

Kramer MO. 1965. Hydrodynamics of the dolphin In Advances in Hydroscience, Vol. 2, V. T. Chow, Ed., New York: Academic Pr. p. 111-130.

Kumph JM, Triantafyllou MS. 1998. A fast-starting and maneuvering vehicle, the ROBOPIKE In Proceedings of the International Symposium on Seawater Drag Reduction, J. C. S. Meng, Ed. Newport, RI. p. 485-490.

Landahl MT. 1962. On stability of a laminar incompressible boundary layer over a flexible surface. $\mathcal{F}$ Fluid Mech, 13:609-632.

Lang TG, Daybell DA. 1963. Porpoise performance tests in a seawater tank. Nav Ord. Test Sta Tech. Rep, 3063.

Lang TG. 1966. Hydrodynamic analysis of cetacean performance In Whales, Dolphins and Porpoises, K. S. Norris, Ed., Berkeley: Univ. of California Press. p. 410-432.

Larrabee EE. 1980. The screw propeller. Sci Amer, 243:134-148.

Lauder GV, Long JH. Jr. 1996. Aquatic locomotion: New approaches to invertebrate and vertebrate biomechanics. Amer Zoo, 36:535-536.

Lauder GV. 1991. An evolutionary perspective on the concept of efficiency: How does it evolve? In Efficiency and Economy in Animal Physiology, R. W. Blake, Ed. Cambridge: Cambridge University Pr. p. 169-184.

Letcher JS, Marshall JK, Oliver JC III, Salvesen N. 1987. Stars \& Stripes. Sci Amer, 257:34-40.

Lindstedt SL, Reich TE, Keim P, LaStayo PC. 2002. Do muscles function as adaptable locomotor springs? 7 Exp Biol, 205:2211-2216.

Livezey BC, Humphrey PS. 1983. Mechanics of steaming in steamer-ducks. Auk, 100:485-488.

Marchaj CA. 1964. Sailing Theory and Practice. New York: Dodd, Mead and Co.

Marchaj CA. 1991. Aero-hydrodynamics of sailing. Camden, Maine: International Marine Publishing.

McCutchen CW. 1977. Froude propulsive efficiency of a small fish, measured by wake visualization In Scale Effects in Animal Locomotion. 1977. T. J. Pedley, Ed. London: Academic Pr. p. 339-363.

Miklosovic DS, Murray MM, Howle LE, Fish FE. 2004. Leading edge tubercles delay stall on humpback whale (Megaptera novaeangliae) flippers. Phys Fluids, 16:L39-L42.

Miller D. 1991. Submarines of the World. New York: Orion Books.

Müller UK, van den Heuvel B LE, Stamhuis EJ, Videler JJ. 1997. Fishfoot prints: Morphology and energetics of the wake behind a continuously swimming mullet (Chelon labrosus Risso). 7 Exp Biol, 200:2893-2906.

Murray M, Howle L. 2003. Spring stiffness influence on an oscillating propulsor. F Fluids Struc, 17:915-926.

Nakashima M, Ono K. 1999. Experimental study of two-joint dolphin robot In Eleventh International Symposium on Unmanned Untethered Submersible Technology. 1999. Lee, NH: Autonomous Undersea Systems Institute. p. 211-218.

Neuhaus PD, O'Sullivan MO, Eaton D, Carff J, Pratt JE. 2004. Concept designs for underwater swimming exoskeletons. Proc 2004 IEEE Internat Conf Robot Autom, 2004:4893-4898.
Pabst DA. 1996. Springs in swimming animals. Amer Zool, 36:723-735.

Pershin SV, Chernyshov LF, Kozlov LF, Koval AP, Zayets VA. 1976. Patterns in the integuments of fast-swimming fishes. Bionika, 10:3-21.

Peterson C JG. 1925. The motion of whales during swimming. Nature, 116:327-29.

Pettigrew JB. 1893. Animal Locomotion. New York: D. Appleton.

Ponganis PJ, Pierce RW. 1978. Muscle metabolic profiles and fiber-type composition in some marine mammals. Comp Biochem Physiol, 59B:99-102.

Prempraneerach P, Hover FS, Triantafyllou MS. 2003. The effect of chordwise flexibility on the thrust and efficiency of a flapping foil. 13th International Symposium on Unmanned Untethered Submersible Technology. 1999. Lee, NH: Autonomous Undersea Systems Institute.

Reidy LW. 1987. Flat plate drag reduction in a water tunnel using riblets. NOSC Tech Rep 1169.

Reif WE, Dinkelacker A. 1982. Hydrodynamics of the squamation in fast swimming sharks. Neues Jahrb Geol Paläotol, 164:184-187.

Reif WE. 1978. Protective and hydrodynamic function of the dermal skeleton of elasmobranchs. Neues Fahrb Geol Paläotol, 157:133-141.

Reif WE. 1985. Morphology and hydrodynamic effects of the scales of fast swimming sharks. Fortsch Zool, 30:483-485.

Riley JJ, Gad-el-Hak M, Metcalfe RW. 1988. Compliant coatings. Ann Rev Fluid Mech, 20:393-420.

Saunders HE. 1951. Some interesting aspects of fish propulsion. Soc Nav Arch. Mar Eng, Ches Sect, 3 May 1951.

Saunders HE. 1957. Hydrodynamics in Ship Design. New York: Soc Nav Arch Mar Eng.

Shaw WC. 1959. Sea animals and torpedoes. NOTS TP 2299, NAVORD Rept. 6573, 1959.

Sokolov V, Bulina I, Rodionov V. 1969. Interaction of dolphin epidermis with flow boundary layer. Nature, 222:267-268.

Stevens GA. 1950. Swimming of dolphins. Sci Prog, 38:524-525.

Suzuki A, Tsuchiya T, Takahashi Y, Tamate H. 1983. Histochemical properties of myofibers in longissimus muscle of common dolphins (Delphinus delphis). Acta Histochem Cytochem, 16:223-231.

Taubes G. 2000. Biologists and engineers create a new generation of robots that imitate life. Science, 288:80-83.

Triantafyllou GS, Triantafyllou MS. 1995. An efficient swimming machine. Sci Amer, 272:64-70.

Triantafyllou GS, Triantafyllou MS, Grosenbaugh MA. 1993. Optimal thrust development in oscillating foils with application to fish propulsion. F Fluids Struct, 7:205-224.

Triantafyllou MS, Barrett DS, Yue D KP, Anderson JM, Grosenbaugh MA, Streitlien K, Triantafyllou GS. 1996. A new paradigm of propulsion and maneuvering for marine vehicles. SNAME Trans, 104:81-100.

Vincent J. 1990. Structural biomaterials. Princeton: Princeton University Press.

Vogel S. 1994. Life in moving fluids. Princeton: Princeton University Press.

Vogel S. 1998. Cat's paws and catapults. New York: W. W. Norton.

Wainwright SA, Pabst DA, Brodie PF. 1985. Form and possible function of the collagen layer underlying cetacean blubber. Amer. Zool., 25:146A.

Walsh MJ. 1990. Riblets. Prog Astro Aero, 123:203-261. 
Watts P, Fish FE. 2001. The influence of passive, leading edge tubercles on wing performance In Proceedings of the Twelfth International Symposium on Unmanned Untethered Submersible Technology. Durham New Hampshire: Autonomous Undersea Systems Institute.

Webb PW. 1975. Hydrodynamics and energetics of fish propulsion. Bull Fish Res Bd Can, 190:1-158.

Webb PW. 1988. Simple physical principles and vertebrate aquatic locomotion. Amer Zool, 28:709-725.

Webb PW. 1997. Designs for stability and maneuverability in aquatic vertebrates: What can we learn In Tenth International Symposium on Unmanned Untethered Submersible Technology. Durham, NH, 1997, pp. 85-108.

Weihs D. 1972. Semi-infinite vortex trails, and their relation to oscillating airfoils. F Fluid Mech, 54:679-690.

Weis-Fogh T, Alexander R McN. 1977. The sustained power output from striated muscle In Scale Effects in Animal Locomotion. 1977. T. J. Pedley, Ed., London: Academic Pr. p. 511-525.
Williams TM, Friedl WA, Fong ML, Yamada RM, Sedivy P, Haun JE. 1992. Travel at low energetic cost by swimming and wave-riding bottlenose dolphins. Nature, 355:821823.

Withers PC, Timko PL. 1977. The significance of ground effect to the aerodynamic cost of flight and energetics of the black skimmer (Rhyncops nigra). 7 Exp Biol, 70:13-26.

Wolfgang MJ, Anderson JM, Grosenbaugh MA, Yue DKP, Triantafyllou MS. 1999. Near-body flow dynamics in swimming fish. 7 Exp Biol, 202:2303-2327.

Wolfgang MJ, Tolkoff SW, Techet AH, Barrett DS, Triantafyllou MS, Yue D KP, Hover FS, Grosenbaugh MA, McGillis WR. 1998. Drag reduction and turbulence control in swimming fish-like bodies In Proceedings of the International Symposium on Seawater Drag Reduction. 1998. J. C. S. Meng, Ed. Newport, RI. p. 485-490.

Yurchenko NF, Babenko VV. 1980. Stabilization of the longitudinal vortices by skin integuments of dolphins. Biophysics, 25:309-315. 

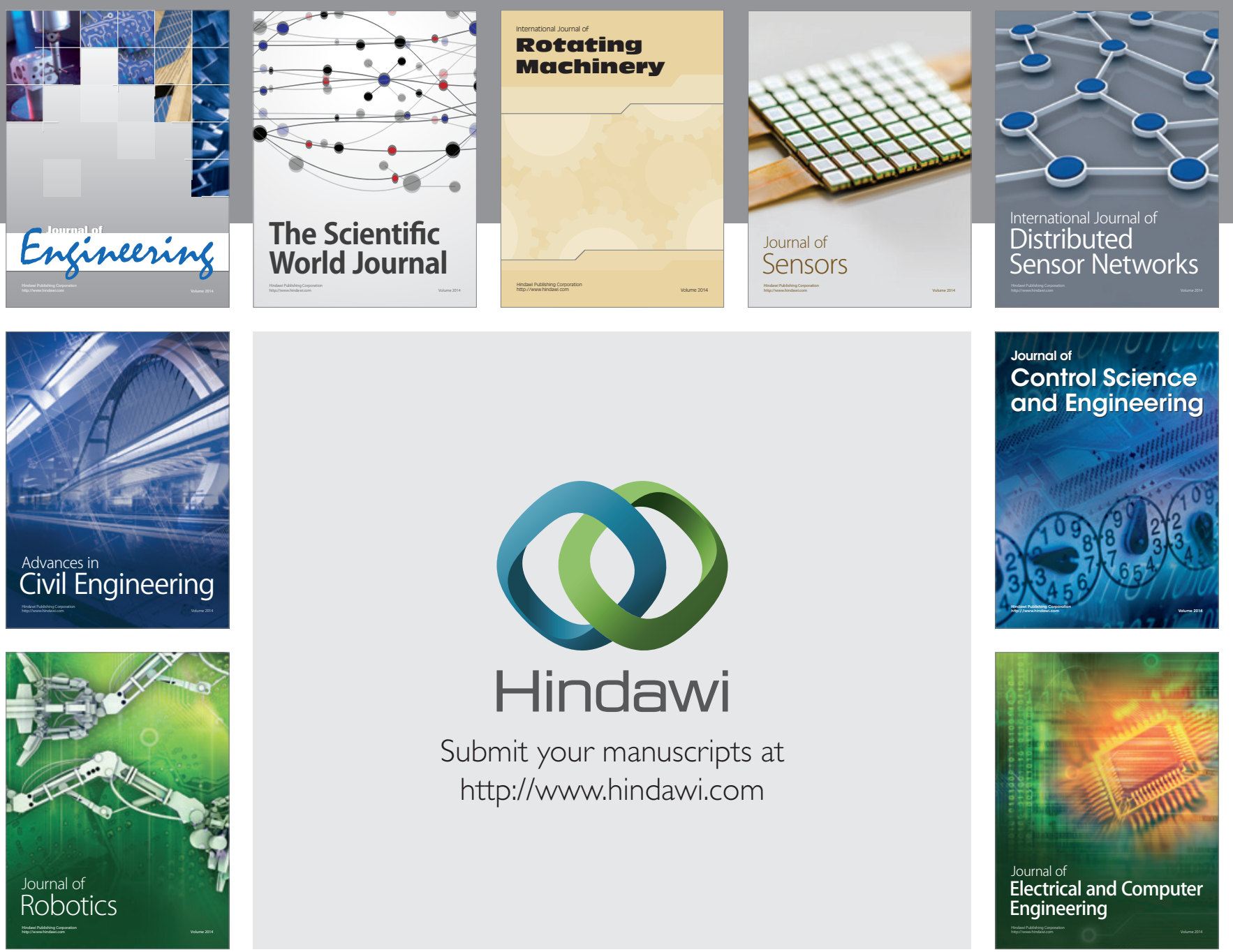

Submit your manuscripts at

http://www.hindawi.com
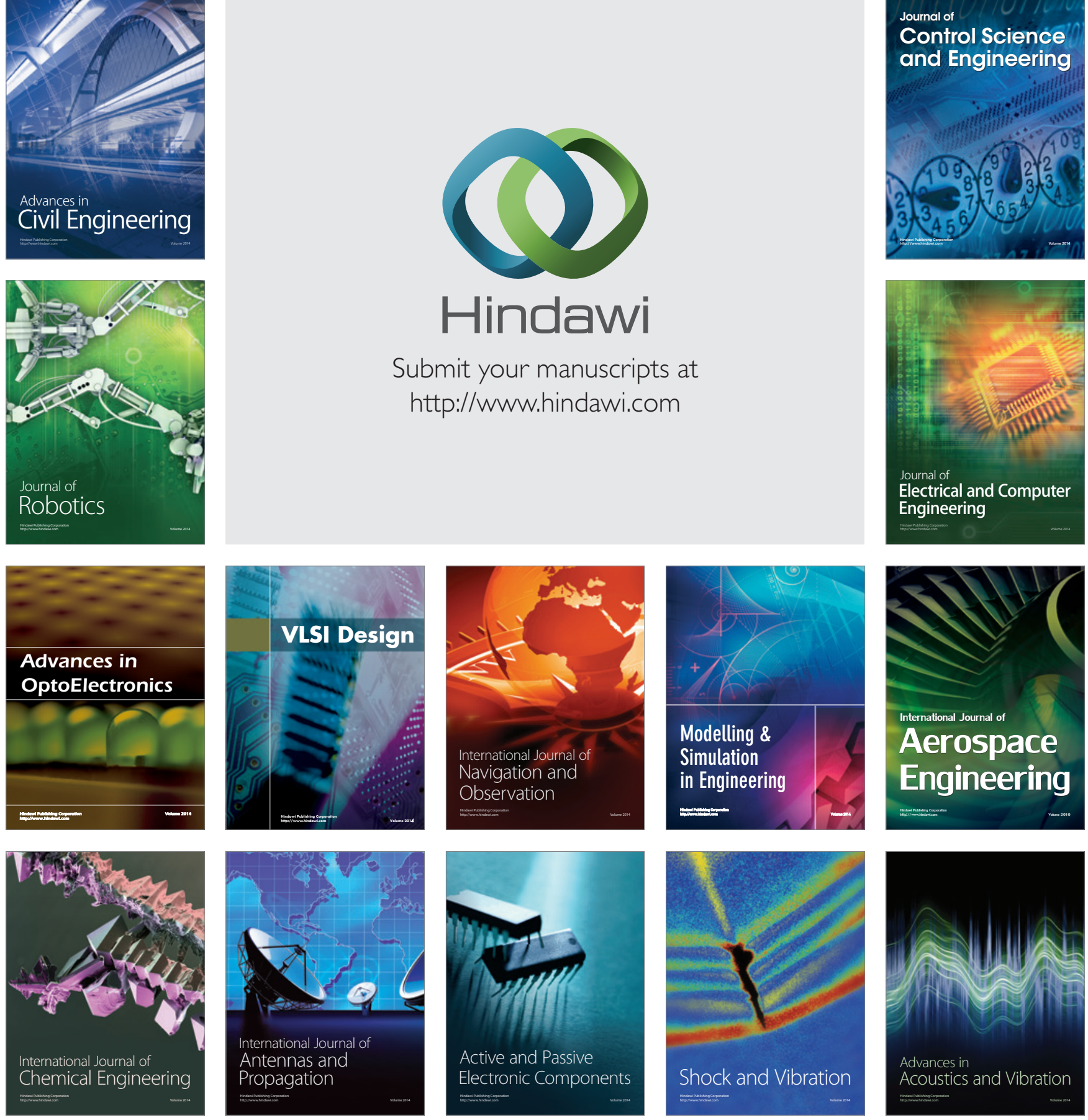